\title{
Acute Cerebellar Ataxia-Uncommon Manifestation of Typhoid Fever
}

\author{
Kiran Kumar1*, Mohammed Khalid1, Shaikh Altaf Basha1, Alyaa Fadhil² \\ ${ }^{1}$ Department of Internal Medicine, GMC Hospital and Research Centre, Ajman, UAE \\ ${ }^{2}$ Department of Clinical Pharmacy, GMC Hospital and Research Centre, Ajman, UAE \\ Email: ${ }^{*}$ drkiran k@yhoo.com
}

Received 9 March 2015; accepted 12 April 2015; published 16 April 2015

Copyright @ 2015 by authors and Scientific Research Publishing Inc.

This work is licensed under the Creative Commons Attribution International License (CC BY).

http://creativecommons.org/licenses/by/4.0/

c) (i) Open Access

\begin{abstract}
Enteric fever is widely prevalent in the tropics. Central nervous system involvement is not rare and reported incidence varies from 5\% to 35\% [1]. Various well-known neuro-psychiatric manifestations include confusional state, encephalopathy, meningism, convulsions and focal neurological deficits. Acute cerebellar ataxia as an isolated neurological complication of enteric fever is very rare and limited to only a few case reports [2]. Here we report a case of enteric fever who presents Acute Cerebellar Ataxia.
\end{abstract}

\section{Keywords}

Enteric Fever, Ataxia, Neurological Manifestations, Typhoid

\section{Introduction}

Enteric fever is a very common infectious condition especially in developing world. It usually presents moderate to high grade fever with or without gastrointestinal manifestations. Diagnosis is usually through blood culture, the first week of illness. However, empirical use of antibiotics may render cultures inconclusive. Therefore, a high index of suspicion is necessary in dealing with cases of fever. Although early diagnosis and treatment prevent complications, delays do occur due to other febrile illness mimicking enteric fever. Such patients can develop various complications including neurological ones. The commonest neurological complications are Encephalopathy and neuro-psychiatric, which occur in patients in whom treatment is delayed. In this case report, we present a case of enteric fever who presents to us with Acute Cerebellar Ataxia.

\section{Case Report}

An 18-year-old male, was admitted with high grade continuous fever for 10 days. He also had associated dry "Corresponding author. 
cough along with fever. Two days prior to presentation, he developed unsteadiness of gait with difficulty in walking. It gradually progressed and within 24 hours, he was unable to walk without support. Along with gait abnormality, he also complained of slurring of speech. There was no altered consciousness, no weakness of extremities and no bowel or bladder abnormalities. Twenty-four hours prior to admission to our hospital, he was shown to a nearby hospital and was started on IV antibiotics (Ceftriaxone and Amikacin).

On general examination, patient had toxic look with temperature of 103 degree Fahrenheit. Heart rate was 78/minute and Blood Pressure was 90/60 mm of Hg. On abdominal examination, spleen was just palpable. It was soft in consistency. Chest was clear. On nervous system examination, his higher mental functions and cranial nerve examination were normal. There were no meningeal signs. There was no sensory disturbance or motor weakness. Patient had bilateral cerebellar signs in form of gait ataxia, finger-nose-finger incoordination, dysdiadochokinesia, dysarthria and truncal ataxia. The tendon jerkes were normal and Babinsky sign was absent.

Hemoglobin was normal. WBC counts were 3000 with $83 \%$ polymorphs. Platelets count was 60,000 at presentation. Liver functions revealed 4 - 5 times elevation of SGOT and SGPT with normal bilirubin. Kidney function tests were normal. Blood culture did not grow any organism. MRI scan of brain with contrast was normal. Lumbar puncture was with held in view of low platelets. WIDAL was strongly positive with $\mathrm{O}-1: 320$ and $\mathrm{H}-$ 1:80.

Patient was treated with intravenous (IV) Ceftriaxone 2 gm twice a day. He became afebrile within 48 hours. Ataxia gradually improved in next 3 - 4 days and at discharge he had minimal ataxia with difficulty in tandem walking. IV Ceftriaxone was continued for a total of 10 days. On follow up after 2 weeks, patient had completely recovered.

\section{Discussion}

Enteric fever is one of the commonest infections seen in our clinical practice. Neuropsychiatric manifestations are encountered in $10 \%-40 \%$ of cases [1] [2]. CNS manifestations secondary to "enteric encephalopathy" is a well-established entity, with a steady increase in neuropsychiatric complications recorded in the last few years; in some patients these neuropsychiatric symptoms dominate the clinical picture [2] [3]. These manifestations include: confusion, delirium, semi-coma, coma, meningism, mutism, dysarthria, acute toxic psychosis, Parkinsonian rigidity, hemiplegia, cerebellar ataxia, myopathy, generalised myoclonus, catatonic schizophrenia, polyneuropathy, hypomania, encephalomyelitis, GB syndrome and transverse myelitis [2] [3].

Cerebellar signs are most likely to appear during the second week of illness, as in our case, but may appear earlier. Sawhney et al. (1988) observed cerebellar involvement in 3 patients on 2nd or 3rd day of enteric fever. It progressed for 1 - 2 days, and then symptoms remained static for 1 - 2 weeks. Patients showed gradual recovery in 1 - 2 weeks. The major cerebellar symptom was gait ataxia with lesser degree of extremity ataxia [4]. Wadia et al. in 1985 described 28 cases of enteric fever with cerebellar ataxia with $25 \%$ incidence in the 1st week and $60 \%$ incidence in the 2nd week [5]. Other workers found an incidence of $19 \%-44 \%$ with a mean onset of 14.8 days $(7$ - 29) for cerebellar ataxia [6].

Why these neuropsychiatric manifestations occur, is still not clear. Certain possible and logical mechanisms are hypothesized to explain these complications, e.g., metabolic disturbances, hyperpyrexia, dehydration and electrolyte imbalance, toxemia and nonspecific cerebral changes in the form of edema and haemorrhage [7]. A cascade of various pathological processes in the cerebrum leads to an acute diffuse encephalomyelitis, causing enteric fever encephalopathy [5].

CT and MRI studies in these cases will be, by and large, normal-indicating that there is no gross structural damage. Blood culture was sterile in our case as patient was on antibiotics prior to admission. Only medical management with antibiotics, preferably after testing for microbial sensitivity will help to alleviate the neurological complications. Recovery from cerebellar ataxia can take many days to few weeks but averages around 7 10 days. Our patient recovered with 5 days of the start on antibiotics.

\section{Conclusion}

Enteric fever is quite common in developing countries and is associated with various neurologic manifestations. It is however important to remember the unusual manifestations of such a common disease such as typhoid fever. In patients presenting fever and cerebellar ataxia, a possibility of enteric fever must be considered. 


\section{List of All Author Contributions}

Dr. Kiran Kumar: Prepared the Case Summary and did the review of literature. Dr. Mohammed Khalid: Provided help in discussion and references.

\section{Declaration}

I declare that we have not received any financial assistance from any sources for this case report. The case report publication was approved by the patient himself.

\section{References}

[1] Baker, L.H. and Baker, A.B. (1974) Nonviral Forms of Encephalitis. In: Baker, L.H. and Baker, A.B., Eds., Clinical Neurology, Harper \& Row Publishers, Philadelphia, Vol. 2, 12-13.

[2] Osuntokun, B.D., Bademosi, O., Ogunremi, K. and Wright, S.G. (1972) Neuropsychiatric Manifestations of Typhoid Fever in 959 Patients. Archives of Neurology, 27, 7-13. http://dx.doi.org/10.1001/archneur.1972.00490130009002

[3] Khosla, S.W., Srivastava, S.C. and Gupta, S. (1977) Neuropsychiatric Manifestations of Typhoid. The Journal of Tropical Medicine and Hygiene, 88, 93-95.

[4] Sawhney, I.M.S., Prabhakar, S., Dhand, U.K. and Chopra, J.S. (1986) Acute Cerebellar Ataxia in Enteric Fever. Transactions of the Royal Society of Tropical Medicine and Hygiene, 80, 85-86. http://dx.doi.org/10.1016/0035-9203(86)90202-6

[5] Wadia, R.S., Dhadpale, S., Kulkarni, et al. (1994) Neurology of Enteric Fever. Revue Neurologique, 1, 57-65.

[6] Lakhotia, M., et al. (2003) Neurological Manifestations of Enteric Fever. Journal, Indian Academy of Clinical Medicine, 4, 197-199.

[7] Choea, J.L., et al. (1987) Extraintestinal Manifestations of Salmonella Infections. Medicine, 66, 349-353. 